\section{Wenn aus Spucken mehr wird}

Bis is zu 70\% aller gesunden Säuglinge spucken, besonders häufig in den ersten vier Lebensmonaten. Wann aber liegt noch ein physiologischer gastroösophagealer Reflux (GÖR) vor, an welchem Punkt ist die Schwelle zur gastroösophagealen Refluxkrankheit (GÖRK) überschritten und wann handelt es sich um eine Kuhmilchallergie? Den Weg zur Diagnose weisen jetzt neue Leitlinien der European Society of Paediatric Gastroenterology, Hepatology and Nutrition (ESPGHAN) zur GÖRK.

Prof. Dr. Sibylle Koletzko aus München war an der Erstellung beteiligt. Sie erklärt, dass die Symptome einer GÖRK gerade bei Säuglingen und Kleinkindern sehr unspezifisch seien und sich nur schwer von anderen Ursachen abgrenzen ließen. Das Spucken ist zwar charakteristisch, aber weder notwendig noch ausreichend für die Diagnose. Weitere Symptome wie chronischer Husten, Schreien, Gedeih- und Fütterstörungen können ebenso auf andere Erkrankungen hindeuten.

Ein typisches Beispiel dafür ist die Kuhmilchproteinallergie, die sich klinisch nicht von einer GÖRK unterscheiden lässt. Sie kann z.B. zur Verengung des Mageneingangs durch Schwellungen führen. Beide Erkrankungen zeigen die gleiche Symptomatik und treten sogar häufig gemeinsam auf. Ein Pricktest oder der Nachweis von spezifischen IgE-Antikörpern liefern Hinweise, sind aber für Diagnose oder Ausschluss allein nicht ausreichend. Klarheit schafft in der Regel erst eine kuhmilchfreie Eliminationsdiät mit therapeutischen Nahrungen, z. B. mit Aminosäuren-Formula wie Neocate $^{\circledR}$. Die Maßnahme empfiehlt sich insbesondere vor der Durchführung einer invasiven Diagnostik. Auch bei älteren Kindern mit Reflux sollten Nahrungsmittelallergien als Ursache berücksichtigt werden. Solche Allergien können Veränderungen der Speiseröhre in Form der eosinophilen Ösophagitis bedingen. „Auch hier ist die Therapie in Form einer Eliminationsdiät erfolgreich“, betont Koletzko. „Können die verursachenden Nahrungsmittel nicht identifiziert werden, sind topisch wirksame Steroide in Form von Suspensionen einzusetzen."

Als Medikation bei gesicherter GÖRK seien dagegen Protonenpumpeninhibitoren (PPI) das Mittel der Wahl. „Von einer blinden PPI-Gabe nur aufgrund der Anamnese bzw. einer pathologischen pH-Metrie ist aber auf jeden Fall abzuraten. Bei Säuglingen in den ersten drei Lebensmonaten besteht wegen des unreifen Enzysmsystems in der Leber zudem die Gefahr der Überdosierung", warnt Koletzko. Bei Nahrungsmittelallergie ohne GÖRK sollten PPI nicht eingesetzt werden. Durch Anhebung des pH-Werts im Magen unter PPI wird Pepsin nicht aktiviert und somit werden Proteine nicht aufgespalten. Untersuchungen bei Erwachsenen zeigen, dass dies die Entstehung von Sensibilisierung auf Nahrungsmittel begünstigt. red

Nach Informationen von Pfrimmer Nutricia, Erlangen

\section{Sechs neue Allergenkomponenten}

Das Unternehmen Phadia bietet in diesem Jahr sechs neue ImmunoCAP® ${ }^{\circledR}$-Allergene an: $\mathrm{i} 208$, i 209, i 210, f 430, t 226 und $\mathrm{w} 232$. Die rekombinante Komponente i 208 ist das Enzym Phospholipase A2 aus Bienengift ( $r A p i ~ m ~ 1)$ und dient als spezifischer Marker für eine Primärsensibilisierung gegen Bienengift. Bei der rekombinanten Komponente i 209 ( $r$ Ves $v 5$ ) handelt es sich um das Wespengift-spezifische Antigen 5. Die Feldwespen-Allergenkomponente i 210 ( $r$ Pol d 5 ) weist eine primäre Sensibilisierung gegen Feldwespengift nach. Die ebenfalls rekombinante Komponente $\mathrm{f} 430$ (rAct d 8) kann zur Abklärung einer Kiwiallergie eingesetzt werden. Da rAct $d 8$ in die Familie der Bet-v-1-homologen Proteine gehört, kann eine Einschätzung erfolgen, ob eine Birkenpollen-assoziierte Allergie vorliegt. Als Marker für eine Primärsensibilisierung gegen Pollen der Familie Cupressaceae ist die native Allergenkomponente $t 226$ (nCup a 1) hilfreich, insbesondere bei der Indikationsstellung zur Immuntherapie. Die neue Komponente w 232 (nSal k 1) schließlich ist ein Majorallergen des Salzkrauts.

Nach Informationen von Phadia, Freiburg

\section{Cluster mit Turbo}

Das Unternehmen Roxall bietet mit Clustoid $^{\circledR}$ das einzige Präparat zur Hyposensibilisierung an, das speziell für die ClusterImmuntherapie entwickelt wurde. Die darin enthaltenen Cluster-Allergoide werden durch ein Hochpolymerisationsverfahren hergestellt und überzeugen durch ihre Verträglichkeit und Wirksamkeit. Seit Juli 2010 ist für Clustoid ${ }^{\circledR}$ ein neues Titrationsschema freigegeben, mit dem die Erhaltungsdosis noch rascher erreicht werden kann: Statt wie bisher vier Injektionen in einer Woche, reichen dazu jetzt zwei Injektionen innerhalb von 15 Minuten aus. Eine einheitliche Dosierung erleichtert die Handhabung in der Praxis zusätzlich. Auch mit dem Therapiestart muss nicht mehr gewartet werden, bis der Pollenflug vorbei ist - er kann direkt in der Pollensaison erfolgen.

Nach Informationen von Roxall Medizin, Hamburg 